\title{
Cardiopatía congénita no corregida y embarazo: reporte de un caso clínico
}

\author{
Brenda Melissa López Gómez*
}

\section{RESUMEN}

En países occidentales, la mayor parte de las cardiopatías en mujeres embarazadas son de tipo congénito, situación que se encuentra relacionada con los importantes adelantos registrados en los últimos años en el ámbito de la cirugía de cardiopatías congénitas. Sin embargo, en países en desarrollo, la situación es diferente: la mayoría de estos niños recibe inadecuado o ningún tipo de tratamiento y llega incluso a la edad adulta sin un diagnóstico establecido; se solicita atención médica cuando la enfermedad se encuentra ya en una etapa avanzada, o bien, cuando una situación concomitante descompensa la cardiopatía. Realizar el diagnóstico y ofrecer un tratamiento adecuado bajo estas circunstancias representa todo un reto para el médico intensivista, sobre todo si se trata de una mujer embarazada, debido a que los cambios propios del embarazo y el posible daño a la integridad del producto dificultan aún más su atención. El objetivo de este artículo es el análisis de las cardiopatías congénitas no corregidas quirúrgicamente más frecuentes en mujeres embarazadas, así como las repercusiones que los cambios fisiológicos inducidos por el embarazo tienen en su evolución, mediante la revisión de un caso clínico de una paciente obstétrica que no se conocía portadora de esta enfermedad.

Palabras clave: Cardiopatía congénita, embarazo.

\section{SUMMARY}

In Western countries, most heart diseases in pregnant women are congenital, a situation that is related to the important advances registered in recent years in the field of congenital heart surgery. However, in developing countries, the situation is different: about $90 \%$ of these children receive inadequate or no treatment at all, and even reach adulthood without an established diagnosis; medical attention is requested when the disease is already at an advanced stage, or when a concomitant situation decompensates the heart disease. Diagnosing and offering an adequate treatment under these circumstances represents a challenge for the intensivist doctor, especially in the case of pregnant women, because the changes inherent to pregnancy and the possible damage to the integrity of the product make their care even more difficult. The aim of this article is the analysis of the most frequent congenital heart diseases not corrected surgically in pregnant women, as well as the repercussions that the physiological changes induced by pregnancy have in their evolution, through the review of a case of an obstetric patient who was not aware that she carried this disease.

Key words: Congenital heart disease, pregnancy.

\section{RESUMO}

Nos países ocidentais, a maioria das cardiopatias em gestantes é de tipo congênita, situação que está relacionada aos importantes avanços registrados nos últimos anos no campo da cirurgia cardíaca congênita. No entanto, nos países em desenvolvimento a situação é diferente, a maioria dessas crianças recebe tratamento inadequado ou nenhum tipo de tratamento, chegando a atingir a idade adulta sem diagnóstico estabelecido, solicitando atendimento médico quando a doença já se encontra em estágio avançado ou quando uma situação concomitante descompensa a cardiopatia. Realizar o diagnóstico e oferecer um tratamento adequado nessas circunstâncias representa um desafio para o médico da unidade de terapia Intensiva, principalmente se for uma gestante, pois as mudanças inerentes à gestação e os possíveis danos à integridade do produto tornam ainda mais difícil sua atenção. $O$ objetivo deste artigo é a análise das cardiopatias congênitas não corrigidas cirurgicamente más frequêntes em gestantes, bem como as repercussões que as alterações fisiológicas induzidas pela gestação têm em sua evolução, através da revisão de um caso clínico de uma paciente obstétrica que não sabia que era portadora dessa doença.

Palavras-chave: Cardiopatia congênita, gestação.

\footnotetext{
* Hospital General de Zona Núm. 1, Instituto Mexicano del Seguro Social, Tapa-
} chula, Chiapas.

Recepción: 23/05/2018. Aceptación: 29/11/2018.

Este artículo puede ser consultado en versión completa en http://www.medigraphic.com/medicinacritica

\section{PRESENTACIÓN DEL CASO}

Mujer de 23 años de edad, sin padecimientos crónicos conocidos; acudió a su unidad médica con embarazo de término para la realización de una cesárea programada por producto en presentación pélvica, la cual se hizo sin complicaciones. Dos días posteriores a la cirugía presentó dificultad respiratoria, taquicardia sinusal e hipertensión arterial. Por disminución en la saturación oxígeno por pulsioximetría $\left(\mathrm{SpO}_{2}\right)$, se intubó; en seguida presentó asistolia, por lo que se iniciaron maniobras de reanimación cardiopulmonar avanzada. Se obtuvo retorno a la circulación al primer ciclo de reanimación; más adelante, presentó una crisis convulsiva tónicoclónica generalizada de dos minutos de duración. Se trasladó a un hospital de segundo nivel, al área de terapia intensiva. El examen físico no reveló déficit neurológico, los campos pulmonares con estertores crepitantes en forma bilateral y los ruidos cardiacos con soplo continuo III/IV audible mayormente en foco pulmonar con reforzamiento del segundo ruido. El monitoreo no invasivo reportó $\mathrm{SpO}_{2}$ de $80 \%$, hipotensión arterial con tensión arterial media (TAM) de $54 \mathrm{mmHg}$ y taquicardia sinusal de 130 por minuto. Los análisis de laboratorio mostraron incremento en el nivel de creatinina de 0.8 a $2.1 \mathrm{mg} / \mathrm{dL}, \mathrm{DHL} 410 \mathrm{UI} / \mathrm{L}, \mathrm{Na} 130 \mathrm{mEq} / \mathrm{L}, \mathrm{Mg} 5.8 \mathrm{meq} / \mathrm{L}$, $\mathrm{Hb} 10.6 \mathrm{~g} / \mathrm{dL}$, leucocitosis $22,200 / \mu \mathrm{L}$ con $93 \%$ de neutrófilos; el resto, normal. El examen general de orina fue normal. La radiografía de tórax portátil se muestra en la Figura 1. El electrocardiograma (ECG) mostró taquicardia sinusal, bloqueo incompleto de la rama derecha del haz de His y crecimiento biventricular.

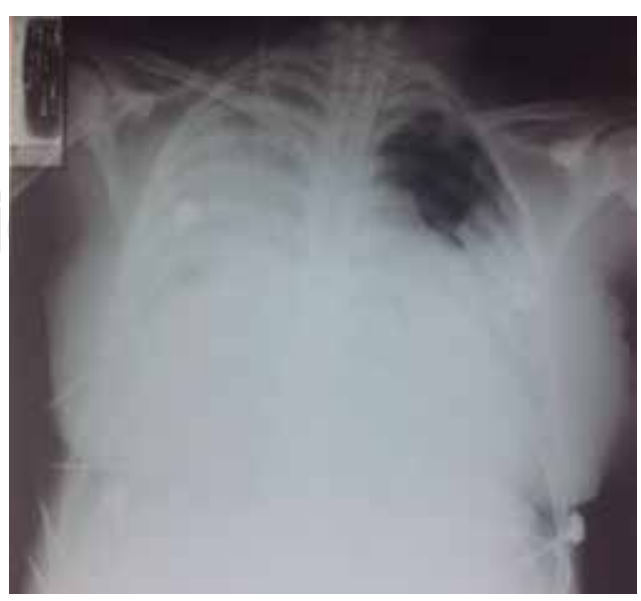

Figura 1: Radiografía de tórax portátil al ingreso a la UCI. 
Se sospechó que la paciente era portadora de una cardiopatía congénita tipo CAP (conducto arterioso persistente) que se descompensó por el embarazo. Se manejó con sedación, analgesia, infusión de norepinefrina y ventilación mecánica con medidas de protección pulmonar; requirió presión positiva al final de la espiración (PEEP) de $12 \mathrm{cmH}_{2} \mathrm{O}$; se utilizó diurético de asa para conseguir balances hídricos negativos. Presentó mejoría gradual y al cuarto día se extubó en forma exitosa. Los niveles de azoados seguían en aumento; se realizaron dos sesiones de hemodiálisis, se resolvió la sobrecarga hídrica y los azoados se normalizaron. Se egresó en buenas condiciones (radiografía de tórax al egreso, Figura 2). El ecocardiograma transtorácico (ECOTT) reportó: 1) cardiopatía congénita acianógena con flujo pulmonar aumentado tipo ventana aortopulmonar, 2) defecto del septum auricular de $4 \mathrm{~mm}$ flujo sistémico-pulmonar, 3) cardiopatía hipertrófica/dilatada, 4) función sistólica moderadamente disminuida con fracción de eyección del ventrículo izquierdo (FEVI) de 44\%,5) hipertrofia ventricular derecha, 6) insuficiencia tricuspídea ligera, 7) insuficiencia pulmonar ligera (Figura 3).

\section{CAMBIOS HEMODINÁMICOS DURANTE EL EMBARAZO}

Los cambios hemodinámicos comienzan en una etapa temprana del primer trimestre. El volumen plasmático empieza a incrementarse durante la sexta semana y se sitúa $50 \%$ por encima del valor inicial hacia el segundo trimestre; a partir de entonces tiende a estabilizarse hasta el momento del periodo expulsivo. Este aumento se sigue de una elevación ligeramente inferior de la masa eritrocítica, lo que da lugar a la anemia relativa del embarazo. ${ }^{1} \mathrm{El}$ incremento en el volumen plasmático aumenta el volumen diastólico final del ventrículo izquierdo. Esta elevación en la precarga se debe a un efecto estrogénico, lo que crea más altos niveles circulantes de renina y una mayor retención de sodio y

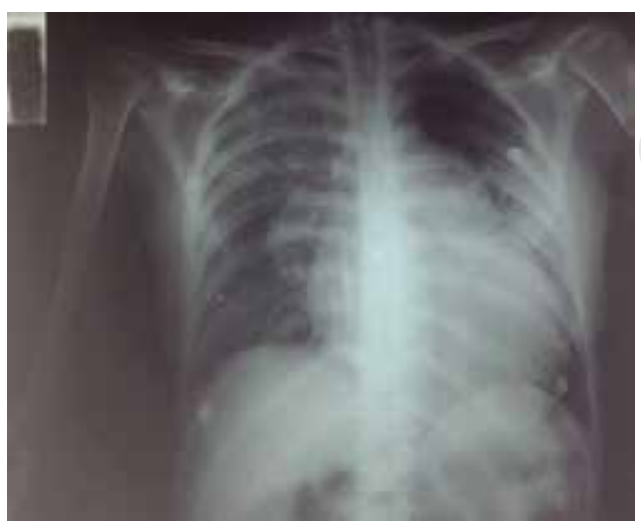

Figura 2: Radiografía de tórax portátil al egreso de la UCI. agua; se han implicado también otras hormonas como la prolactina, el lactógeno placentario humano, las prostaglandinas y la hormona del crecimiento. Se incrementa el volumen sistólico y la frecuencia cardiaca aumenta de $15-20 \%$. El efecto neto de estos cambios es una elevación de $30-50 \%$ en el gasto cardiaco (GC) al final del primer trimestre, llegando a su pico máximo entre el segundo y tercer trimestre. ${ }^{2}$ Estos efectos fisiológicos son facilitados por otra condición: la irrigación uterina se torna más intensa como consecuencia del desarrollo placentario, lo cual produce una disminución de la resistencia vascular sistémica (RVS) que puede originar un ligero descenso de la presión arterial que se inicia en el primer trimestre; ${ }^{1}$ la disminución de la RVS se continúa hasta la semana 32 , cuando la postcarga comienza a incrementar de nuevo. ${ }^{2}$ Finalmente, en el tercer trimestre, la reducción de la precarga podría ocurrir debido a la compresión de la vena cava inferior por el útero grávido, lo que baja el GC. ${ }^{2}$

\section{CAMBIOS HEMODINÁMICOS DURANTE LA LABOR, PARTO Y POSTPARTO}

En esta etapa existe un incremento, inducido por catecolaminas, en la frecuencia cardiaca y el volumen sis-
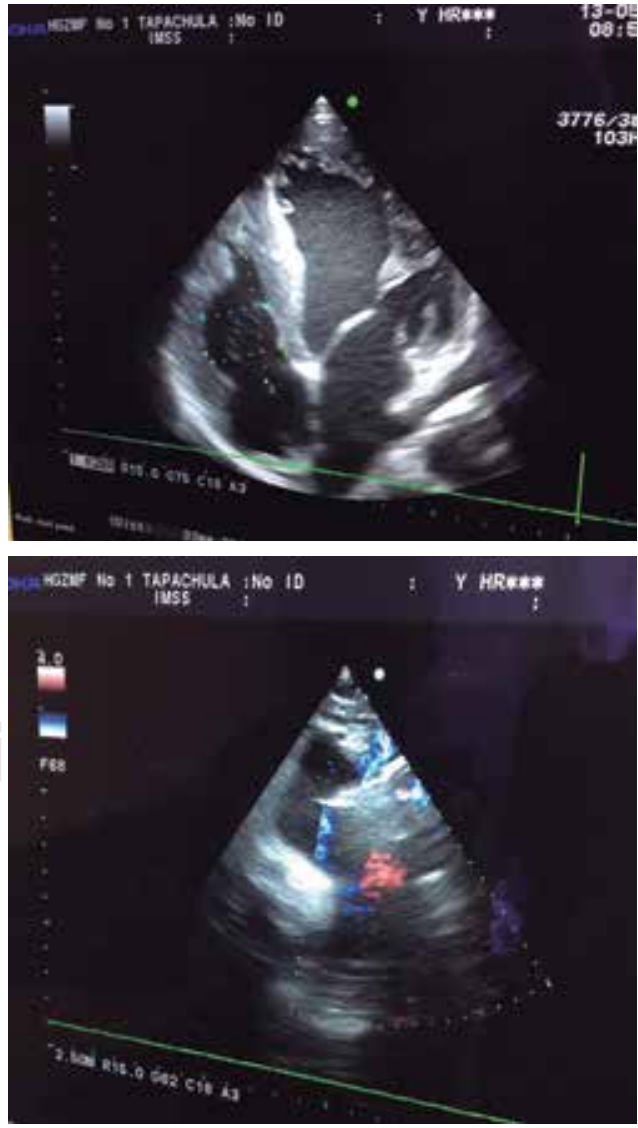

Figura 3: Ecocardiograma transtorácico. 
tólico, debido al dolor y la ansiedad. Durante el periodo periparto puede haber un incremento del GC de hasta $31 \%$ y de hasta $50 \%$ en el segundo estadio de la labor. Cambios abruptos en el balance de líquidos resultan en esta etapa: cada contracción uterina libera entre 300$500 \mathrm{~mL}$ de sangre hacia el torrente circulatorio, lo cual ocasiona aumento súbito del GC y la presión arterial. ${ }^{2}$ Con la expulsión del feto se producen grandes cambios en la hemodinamia, sobre todo dentro de las primeras 12 a 24 horas postparto. Dentro de la primera hora del parto, el GC puede seguir aumentando hasta $80 \%$ por encima de los valores previos a la labor debido a la desaparición de la compresión a la vena cava inferior y a la autotransfusión de sangre desde el útero ${ }^{1,2}$ (se denomina autotransfusión debido a que la contracción sostenida del útero transfiere el volumen sanguíneo contenido en sus plexos venosos hacia la circulación general, lo cual continúa a lo largo de las 24 a 72 horas posteriores al nacimiento). ${ }^{1}$ Parte de este volumen intravascular es perdido en el parto a causa del sangrado que acontece (aproximadamente $400-500 \mathrm{~mL}$ en el parto vaginal y $800-1,000 \mathrm{~mL}$ en cesárea). ${ }^{1,2} \mathrm{~A}$ su vez, puede haber más alteraciones en el estado hemodinámico debido a la pérdida de la resistencia baja de la placenta y a un aumento relativo en la RVS, así como en la movilización del edema dependiente y el fluido intersticial. ${ }^{2}$

\section{CARDIOPATÍAS CONGÉNITAS NO CORREGIDAS EN ADULTOS}

Las cardiopatías congénitas más comunes en las que es posible la supervivencia natural (sin cirugía) hasta la edad reproductiva se enlistan la Tabla $1^{3}$ en orden aproximado de prevalencia en mujeres, y se describen a continuación.

1. Defecto septal auricular (DSA). El subtipo más frecuente es el ostium secundum (75\%) y es debido a la ausencia de tejido en la fosa oval. ${ }^{4}$ Estos defectos crean un cortocircuito de izquierda a derecha cuyo grado depende del tamaño del defecto, la distensibilidad del ventrículo derecho (VD) e izquierdo (VI) y la resistencia vascular pulmonar (RVP) versus la RVS.

\section{Tabla 1: Malformaciones acianóticas y cianóticas comunes con expectativa de supervivencia adulta, en orden descendente de prevalencia en mujeres.}

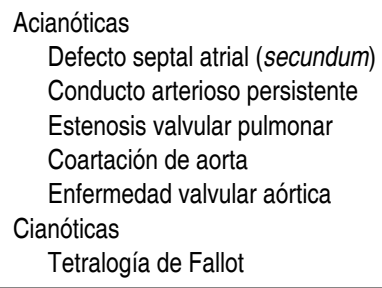

Tomado de: Pitkin RM, Perloff JK, Koos BJ, Beall MH. Pregnancy and congenital heart disease. Ann Intern Med. 1990;112:445-454.
Por lo tanto, cuando las cuatro cámaras se encuentran en libre comunicación durante la diástole, la sangre de la aurícula izquierda (Al) es desviada a la aurícula derecha (AD), causando incremento del flujo sanguíneo y dilatación gradual de la AD, el VD y las arterias pulmonares. Como resultado del flujo pulmonar aumentado, la presión sistólica del VD puede estar elevada; sin embargo, por lo general, la RVP es normal. ${ }^{5} \mathrm{La}$ hipertensión pulmonar (HTP) debido a la exposición crónica al alto flujo pulmonar, así como el síndrome de Eisenmenger, es rara en este tipo de defectos y, por lo general, se presenta a edad avanzada. ${ }^{3}$ En el examen físico, los hallazgos incluyen un soplo mesosistólico en el borde esternal izquierdo, desdoblamiento amplio y fijo del segundo ruido pulmonar, impulso del VD palpable y sostenido y, en ocasiones, un retumbo diastólico en el borde esternal inferior izquierdo. ${ }^{4}$ Debido al VD agrandado, el ECG muestra típicamente un patrón de bloqueo incompleto de la rama derecha junto con una desviación del eje a la derecha en ostium secundum o a la izquierda en ostium primum. ${ }^{4}$ La gestación es bien tolerada en pacientes con defectos no reparados sin HTP significativa debido a que el efecto del incremento del volumen plasmático y el GC inducido por el embarazo en un VD con sobrecarga previa de volumen es contrarrestado por el descenso de la RVS. ${ }^{6,7}$ Sin embargo, las arritmias y disfunción ventricular pueden ocurrir, sobre todo si el defecto es grande. La posibilidad de embolización paradójica aumenta cuando la vasodilatación sistémica y/o la elevación de la RVP ocasionan un cambio transitorio del cortocircuito de derecha a izquierda. ${ }^{7}$

2. Conducto arterioso persistente (CAP). En el feto, el conducto arterioso conecta la porción proximal de la arteria pulmonar izquierda con la porción proximal de la aorta descendente justo por debajo de la entrada de la arteria subclavia izquierda. Desde una perspectiva clínica, el CAP en el adulto puede ser clasificado de la siguiente forma: ${ }^{4}$ 1) Silente: CAP diminuto detectado solo por medios no clínicos (usualmente ecocardiografía), soplo cardiaco no audible; 2) Pequeño: soplo continuo infraclavicular izquierdo audible irradiado hacia la parte superior izquierda de la espalda, cortocircuito izquierda-derecha, cambios hemodinámicos poco significativos; 3) Moderado: soplo continuo audible, agrandamiento de la Al y VI con algún grado de hipertensión pulmonar; 4) Grande: es usual que los pacientes adultos tengan fisiología de Eisenmenger, soplo continuo ausente, cortocircuito derecha-izquierda, cianosis diferencial y dedos hipocráticos, manifestaciones a la auscultación de HTP (soplo eyectivo, reforzamiento del segundo ruido y soplo de regurgitación pulmonar de Graham Steele). EI ECG puede ser normal (CAP pequeño), mostrar hipertrofia ventricular izquierda aislada (CAP moderado) o hipertrofia biventricular (CAP 
grande con HTP). Una mujer joven asintomática con un CAP pequeño o moderado y presión arterial pulmonar normal puede esperar un embarazo no complicado. ${ }^{3}$ Con un cortocircuito grande de izquierda a derecha, la disminución en la RVS inducida por el embarazo ayuda a reducir el flujo ductal, pero este efecto puede no compensar toda la carga hemodinámica debida al aumento del volumen plasmático en el embarazo, lo que puede resultar en falla ventricular. La paciente con mayor riesgo es aquella con un CAP no restrictivo, RVP suprasistémica y shunt reverso (síndrome de Eisenmenger), ya que la gestación, al bajar la RVS, ocasiona que se incremente el cortocircuito de derecha a izquierda a través del ducto, reduciendo aún más la saturación de oxígeno arterial. ${ }^{3}$

3. Estenosis pulmonar (EP). Es la forma más frecuente de obstrucción del lado derecho. Por lo general, es una anomalía congénita aislada y ocurre en $7-10 \%$ de los pacientes con cardiopatías congénitas. La morfología de la válvula puede ser de tres tipos: en forma de domo (la más común), displásica y bicúspide. ${ }^{4}$ En el examen físico puede haber una onda «a» yugular prominente (debido a la contracción enérgica de la $A D$ para llenar un VD con poca distensibilidad), levantamiento paraesternal palpable deI VD (por hipertrofia del VD), un clic de eyección en el borde esternal superior izquierdo (debido a la apertura abrupta de las valvas o una arteria pulmonar principal dilatada) y un soplo mesosistólico paraesternal en crescendo-decrescendo (por la turbulencia del flujo a través de la válvula estenótica). ${ }^{4}$ El ECG puede ser normal en EP leve o puede haber desviación del eje a la derecha e hipertrofia del VD si es severa. La historia natural de la enfermedad permite la supervivencia hasta la edad adulta, incluso en casos en los que existe una obstrucción significativa al flujo de salida al VD. La EP leve a moderada representa poca o ninguna amenaza para la madre, y en ocasiones, incluso la EP severa es tolerada a pesar de la carga de volumen adicional impuesta por el embarazo en un VD ya sobrecargado de presión. ${ }^{3}$

4. Coartación de aorta. Es la anomalía más común del arco aórtico, representa $8 \%$ de todos los casos de cardiopatías congénitas; $20 \%$ se diagnostican durante la adolescencia o la edad adulta. ${ }^{4}$ La consecuencia fisiológica mayor es un incremento en la postcarga del VI. La presión sanguínea está dramáticamente incrementada en la aorta y las ramas arteriales proximales al sitio de la coartación y está disminuida distal a esta. El flujo sanguíneo de la parte inferior del cuerpo es mantenido por el desarrollo de colaterales en las arterias subclavia, mamaria interna, intercostales y espinal. La hipertensión sistémica es atribuida tanto a una obstrucción mecánica como a un fenómeno de hipoperfusión renal. La palpación simultánea de las arterias braquial y femoral puede detectar un retraso en el pulso femoral. Debe medirse la presión arterial en las cuatro extremidades. Una presión arterial sistólica en los brazos $\geq 20 \mathrm{mmHg}$ sobre la presión de las piernas confirma la sospecha. La auscultación revela un fuerte sonido de cierre aórtico y en pacientes con válvula aórtica bicúspide (VAB) asociada, la auscultación revela un clic aórtico de eyección seguido por un soplo mesosistólico irradiado a la base. Un soplo sistólico suave puede escucharse sobre la espalda, en particular, entre las escápulas. La presencia de vasos colaterales largos puede dar origen a un soplo continuo en la espalda o el abdomen. ${ }^{4}$ EI ECG puede mostrar varios grados de hipertrofia del VI en función de la duración y la gravedad de la coartación o la hipertensión sistémica. La coartación de aorta no reparada conlleva un alto riesgo debido a que los cambios hormonales del embarazo provocan alteraciones en el tejido conectivo arterial sistémico, lo que incrementa el riesgo de ruptura aórtica o disección, así como también el riesgo de hemorragia cerebral secundaria a ruptura de un aneurisma del polígono de Willis. ${ }^{8,9} \mathrm{El}$ riesgo de complicaciones hipertensivas durante el embarazo es más alto, incluyendo hipertensión preexistente, hipertensión inducida por el embarazo, preeclampsia y eclampsia. ${ }^{10}$ Puede presentarse falla ventricular por el aumento del volumen plasmático impuesto a un VI ya sobrecargado de presión, pero no es común. ${ }^{8}$

5. Enfermedad valvular aórtica. En mujeres que contemplan el embarazo, la mayoría de las veces, es de origen congénito, ${ }^{11}$ siendo la más frecuente la VAB. La VAB es un ejemplo clásico de enfermedad cardiaca que de manera usual es asintomática en la infancia, pero que llega a ser sintomática en la edad adulta. ${ }^{4} \mathrm{De}$ bido a la alta susceptibilidad para endocarditis infecciosa, esta anomalía puede detectarse después del parto como fiebre o regurgitación aórtica aguda. ${ }^{3} \mathrm{Al}$ examen físico, se escucha un clic de eyección tanto en el ápex y el borde esternal superior derecho. Esto es seguido por un soplo de eyección sistólico que puede irradiar al cueIlo. El ECG muestra ritmo sinusal y eje QRS normal con datos de hipertrofia del VI. ${ }^{4}$ En las pacientes con VAB, esta válvula puede funcionar con normalidad a lo largo de la vida, puede desarrollar calcificación progresiva y estenosis o regurgitación con o sin infección. ${ }^{12}$ Una $\mathrm{VAB}$ funcionalmente normal a menudo no se reconoce en mujeres jóvenes debido a la mayor prevalencia en varones y los mínimos signos en la auscultación; el embarazo en este escenario no representa un riesgo. En general, la VAB con regurgitación crónica (moderada a severa) se tolera bien en el embarazo, siempre que el VI preserve una función normal. La disminución en la RVS del embarazo junto con el aumento en la frecuencia cardiaca (diástole más corta) provocan disminución en el flujo regurgitante. El riesgo de endocarditis infecciosa es alto, por lo que la profilaxis con antibióticos es 
obligatoria durante la labor y el parto. ${ }^{8}$ En mujeres con VAB estenótica, el incremento en el GC del embarazo tiende a ser tolerado si la estenosis es leve a modera$\mathrm{da}$, pero si es severa (área valvular $<1 \mathrm{~cm}^{2}$; gradiente pico $>75 \mathrm{mmHg}$ o $\mathrm{FEVI}<55 \%$ ), el riesgo es muy significativo. ${ }^{13}$ En una embarazada con estenosis severa, su capacidad limitada para aumentar el GC puede resultar en una elevación anormal de las presiones de llenado y sistólica del VI, que a su vez precipitará falla cardiaca o isquemia. Además, un VI hipertrofiado, con poca distensibilidad, es sensible a una reducción en la precarga. Por ello, la consecuente caída excesiva del GC puede llevar a hipotensión, hipoperfusión, o bien, flujo excesivo con hipoperfusión. ${ }^{7,13}$

6. Tetralogía de Fallot (TF). Es la cardiopatía congénita cianógena más común que permite una supervivencia sin cirugía hasta la edad reproductiva. ${ }^{8}$ Once por ciento sobreviven a los 20 años, $6 \%$ a los 30 años y $3 \%$ a los 40 años. ${ }^{4}$ Se origina debido a que durante el desarrollo cardiaco se produce un desplazamiento anterior y cefálico del septum infundibular, lo que resulta en cuatro características: 1) defecto del septum ventricular (DSV) no restrictivo; 2) cabalgamiento aórtico (de menos de $50 \%$ sobre el VD); 3) obstrucción al tracto de salida del VD, que puede ser infundibular, valvular o, usualmente ambas, con o sin estenosis supravalvular o de las ramas de la arteria pulmonar; 4) hipertrofia del VD. Los cambios hemodinámicos dependen de algunas variables. En presencia de un DSV por lo común grande y no restrictivo, las presiones sistólicas en el VD y VI son iguales. Si la obstrucción del tracto de salida del VD es leve, el cortocircuito a través del DSV puede ser de izquierda a derecha, dando lugar a la llamada «tetralogía acianótica» o «Fallot rosado». En pacientes con TF típica, la obstrucción del tracto de salida del VD es severa, con un cortocircuito de derecha a izquierda, lo que crea un Fallot cianótico clásico. El examen clínico de pacientes no operados puede revelar cianosis digital y peribucal, levantamiento palpable del VD, segundo ruido único (el componente pulmonar no es audible), clic de eyección aórtico (por la aorta cabalgada y dilatada) y soplo sistólico de eyección (causado por el flujo a través del tracto de salida del VD obstruido). EI ECG puede mostrar desviación del eje a la derecha e hipertrofia del VD. ${ }^{4}$ En el embarazo, el descenso de la RVS, junto con el aumento del retorno venoso a un VD obstruido dan como resultado un incremento del cortocircuito de derecha a izquierda y, por tanto, una mayor disminución en la saturación arterial de oxígeno sistémica, cambios que son perjudiciales tanto para la madre como para el feto. Durante la labor y el parto, una caída súbita en la RVS puede precipitar cianosis intensa, síncope y muerte. El alumbramiento puede abrupta y peligrosamente reducir el flujo sanguíneo arterial sistémico. ${ }^{8}$

\section{DISCUSIÓN}

Se describe el caso de una paciente obstétrica que inició en el puerperio inmediato con insuficiencia respiratoria que evolucionó a paro cardiorrespiratorio secundario a la descompensación de una cardiopatía congénita de la cual no se sabía portadora. El diagnóstico fue clínico en un inicio, al detectarse, durante la exploración física, un soplo cardiaco continuo y fuerte en el segundo espacio intercostal izquierdo, el cual no se encuentra dentro de las alteraciones que pueden ser atribuibles a los cambios inducidos por el embarazo; los estudios complementarios, radiografía de tórax y ECG, apoyaron la sospecha clínica de un probable CAP no corregido. EI ECOTT confirmó que se trataba de una cardiopatía congénita poco usual y muy parecida al CAP, la ventana aortopulmonar, asociada con un DSA. Ambas son malformaciones con flujo pulmonar aumentado y de cortocircuito izquierda a derecha. La ventana aortopulmonar consiste en la comunicación entre la aorta ascendente y la arteria pulmonar, con características clínicas y hemodinámicas similares al CAP; sin embargo, se diferencia de este en que la luz de la arteria pulmonar se comunica en forma directa con la aorta sin que exista un ducto y, por lo tanto, las paredes arteriales se encuentran en estrecha aposición. La causa de la descompensación fueron los cambios fisiológicos hemodinámicos que acontecieron en el puerperio, principalmente las alteraciones en el balance de líquidos, que conllevan un aumento de la precarga a causa de la pérdida de compresión de la vena cava inferior, la autotransfusión de sangre desde el útero, la movilización del edema dependiente y del fluido intersticial, así como al aumento relativo en la RVS (debido a que se pierde la resistencia baja de la placenta). Si bien estos cambios acontecen en general en pacientes obstétricas, en este caso no fueron tolerados debido a la enfermedad cardiaca preexistente, resultando en congestión hídrica e incremento del cortocircuito de izquierda a derecha por aumento de la RVS, lo que desencadenó los síntomas de insuficiencia cardiaca congestiva con hipoxemia severa y, al final, choque cardiogénico. El manejo se enfocó en reducir la precarga y mejorar el GC. Debido a que la paciente presentaba inestabilidad hemodinámica, fue tratada con un vasopresor a la dosis más baja para mantener cifras adecuadas de TAM, ya que dosis altas condicionarían mayor elevación de la postcarga, con incremento del cortocircuito izquierda a derecha. En cuanto a la ventilación mecánica, se evitaron presiones de la vía aérea excesivas que condicionarían un aumento importante de la presión arterial pulmonar y, con ello, inversión del cortocircuito. Se administró diurético intravenoso para reducir la congestión pulmonar y requirió hemodiálisis intermitente ante la falta de me- 
joría en la función renal. Evolucionó a la recuperación y fue egresada para continuar su protocolo de estudio.

\section{CONCLUSIONES}

En la actualidad, las cardiopatías congénitas representan a nivel mundial una causa común de morbilidad y mortalidad materna debida a enfermedad cardiaca. Por ello es importante la sospecha de este tipo de padecimientos en pacientes con alteraciones en la exploración cardiológica. La evaluación detallada del tipo específico de cardiopatía, así como el conocimiento de su fisiología y las repercusiones que los cambios inducidos por el embarazo, el parto y el puerperio puedan tener en la hemodinamia son claves para prevenir complicaciones $y$, en caso de que se presenten, establecer un plan de manejo adecuado que mejore el pronóstico tanto de la madre como del producto.

\section{BIBLIOGRAFÍA}

1. Warnes CA. Embarazo y enfermedades cardiovasculares. Zipes D, Libby P, Bonow R, Braunwald E, editores. Braunwald: Tratado de cardiología. 9.a ed. España: Elsevier Saunders; 2013. pp. 1793-1806.

2. Stergiopoulos K, Shiang E, Bench T. Pregnancy in patients with pre-existing cardiomyopathies. J Am Coll Cardiol. 2011;58(4):337-350.

3. Pitkin RM, Perloff JK, Koos BJ, Beall MH. Pregnancy and congenital heart disease. Ann Intern Med. 1990;112:445-454.

4. Wu JC, Child JS. Common congenital heart disorders in adults. Curr Probl Cardiol. 2004;29:641-700.
5. Bhatt AB, DeFaria-Yeh D. Pregnancy and adult congenital heart disease. Cardiol Clin. 2015;33:611-623.

6. Domènech AP, Gatzoulis MA. Embarazo y cardiopatía. Rev Esp Cardiol. 2006;59:971-984.

7. Colman JM, Siu SC. Pregnancy in adult patients with congenital heart disease. Prog Paediatr Cardiol. 2003;17:53-60.

8. Perloff JK. Congenital heart disease and pregnancy. Clin Cardiol. 1994;17:579-587.

9. European Society of Gynecology (ESG); Association for European Paediatric Cardiology (AEPC); German Society for Gender Medicine (DGesGM), Regitz-Zagrosek V, Blomstrom Lundqvist C, Borghi C, et al. ESC Guidelines on the management of cardiovascular diseases during pregnancy: the Task Force on the Management of Cardiovascular Diseases during Pregnancy of the European Society of Cardiology (ESC). Eur Heart J. 2011;32(24):3147-3197.

10. Brickner ME. Cardiovascular management in pregnancy: congenital heart disease. Circulation. 2014;130:273-282.

11. Swan L. Congenital heart disease in pregnancy. Best Pract Res Clin Obstet Gynaecol. 2014;28(4):495-506.

12. Yener N, Oktar GL, Erer D, Yardimci MM, Yener A. Bicuspid aortic valve. Ann Thorac Cardiovasc Surg. 2002;8(5):264-267.

13. Gandhi M, Martin SR. Cardiac disease in pregnancy. Obstet Gynecol Clin North Am. 2015;42(2):315-333.

Patrocinios y conflicto de intereses: La autora declara que no tiene ningún conflicto de intereses (económi$\mathrm{co}$, profesional o personal) real, potencial o potencialmente percibido que pueda resultar en un sesgo en la publicación de este trabajo.

Correspondencia:

Brenda Melissa López Gómez

Hospital General de Zona Núm. 1, IMSS.

Carretera Costera y Anillo Periférico s/n,

Col. Centro, Tapachula, Chiapas, México.

E-mail: bmeliss@hotmail.com 